\title{
Kinobead/LC-MS phosphokinome profiling enables rapid analyses of kinase-dependent cell signaling networks
}

Martin Golkowski, ${ }^{1 *}$ Venkata Narayana Vidadala, ${ }^{2}$ Ho-Tak Lau, ${ }^{1}$ Anna Shoemaker, ${ }^{1}$ Masami Shimizu-Albergine, ${ }^{3}$ Joseph Beavo, ${ }^{1}$ Dustin J. Maly, ${ }^{2}$ and Shao-En Ong. ${ }^{1 *}$

${ }^{1}$ Department of Pharmacology, University of Washington, Seattle, WA 98195, USA

${ }^{2}$ Department of Chemistry, University of Washington, Seattle, WA 98195, USA

${ }^{3}$ University of Washington Medicine Diabetes Institute, University of Washington, Seattle, WA 98109, USA

*Correspondence: shaoen@u.washington.edu (S-E.O.), golkom@uw.edu (M.G.)

\section{SUPPLEMENTARY INFORMATION}

S1: Title Page

S2: Figure S1. Structure of the seven kinobead reagents used in this study.

S3: Figure S2. Serial enrichment of kinases from cell lysates using kinobeads

S4: Figure S3. Testing the performance of our kinobead/LC-MS workflow

S5: Figure S4. Comparing the performance of kinobead/LC-MS phosphoproteomics and global phosphoproteomics in MA10 cells

S6. Supplementary Table S1. Protein and peptide quantification data, secondary analyses. 

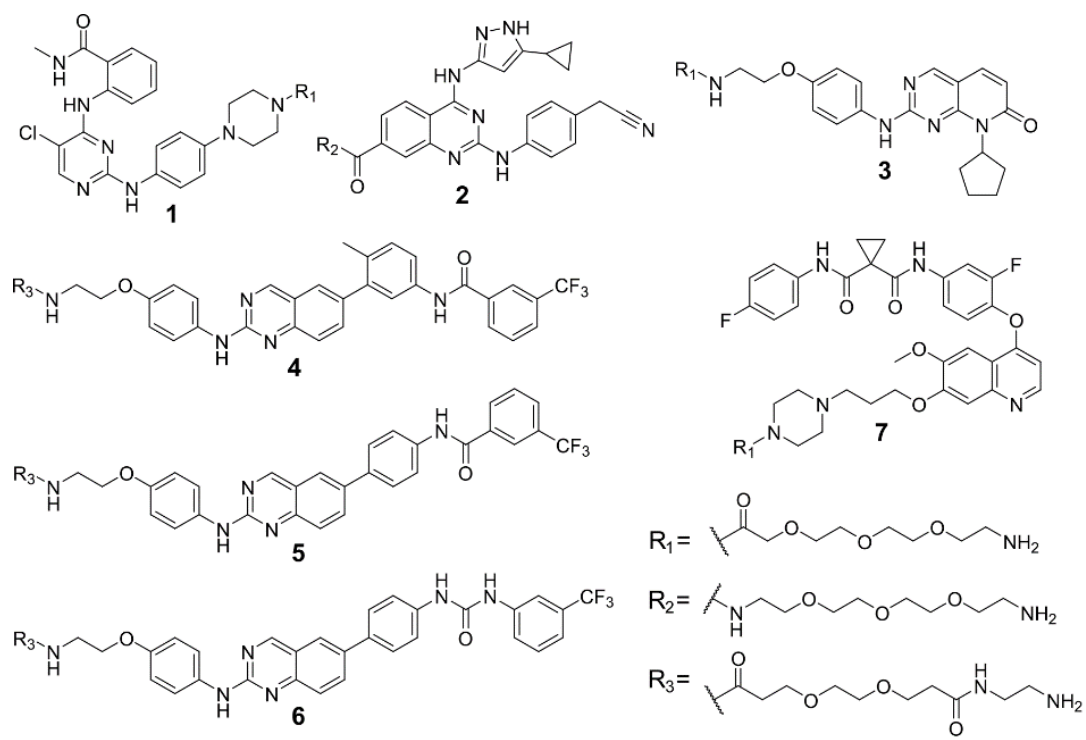

Figure S1. Structure of the seven kinobead reagents used in this study. For a detailed description of synthetic procedures and compound characterization see Golkowski et al. 2017.22 

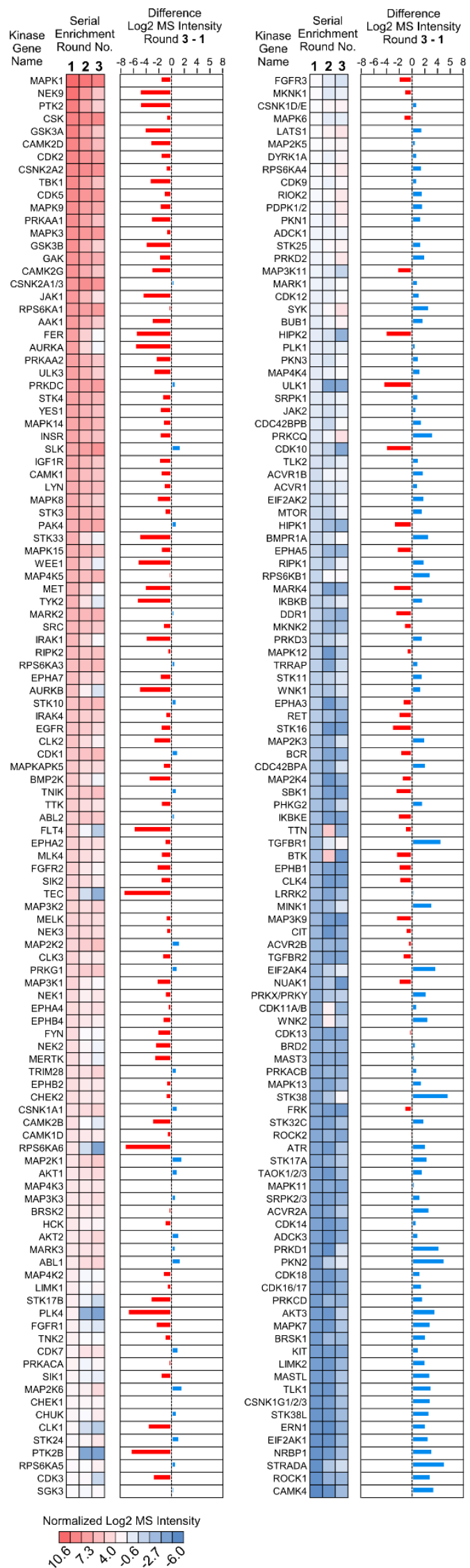

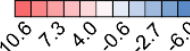

Figure S2. Serial enrichment of kinases from cell lysates using kinobeads. Cell lysates were subjected to three rounds of affinity enrichment with kinobeads and each kinobead pulldown analyzed separately by LC-MS (see aso 'Experimental Procedures'). The results show that many highly abundant kinases are depleted during early rounds of affinity enrichment, whereas other low abundance kinases are preferable enriched in later rounds. 

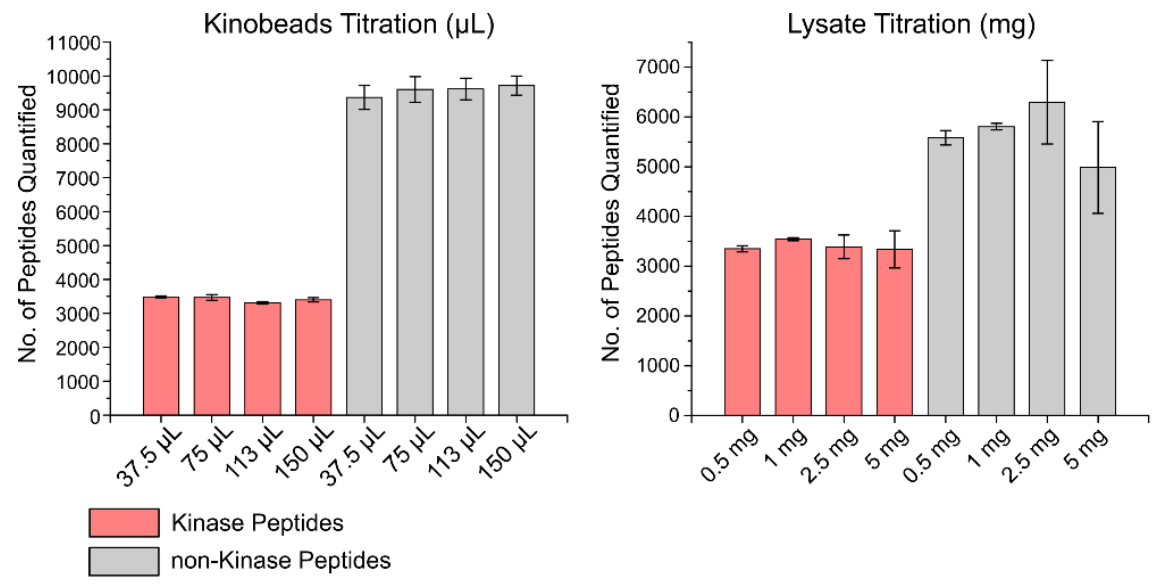

Figure S3. Testing the performance of our kinobead/LC-MS workflow. Kinobead/LC-MS quantification of peptides (kinases and non-kinases) in HeLa cell lysate using varying amounts of kinobeads and $1 \mathrm{mg}$ protein sample (left panel) or varying amounts of protein sample and $100 \mu \mathrm{L}$ kinobeads (right panel). Bars represent the mean of three biological replicates, error bars are the S.D. Data were obtained in single analytical LC-MS runs on a Thermo Scientific Orbitrap Elite mass spectrometer. 
Comparison Global vs. Kinobead-MS

Phosphoproteomics

All Quantified Phosphosites

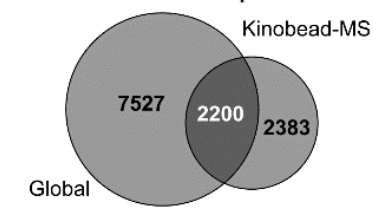

Quantified Kinase Phosphosites

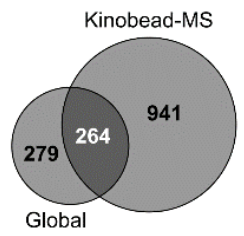

Figure S4. Comparing the performance of kinobead/LC-MS phosphoproteomics and global phosphoproteomics in MA10 cells. The results indicate that kinobead/LC-MS phosphoproteomics identifies 3- to 4-fold more kinase phosphopeptides than global phosphoproteomics using a similar experimental setup (see 'Experimental procedures' and Tables S1). 